\title{
Tasa de infiltración de agua en suelos agrícolas.
}

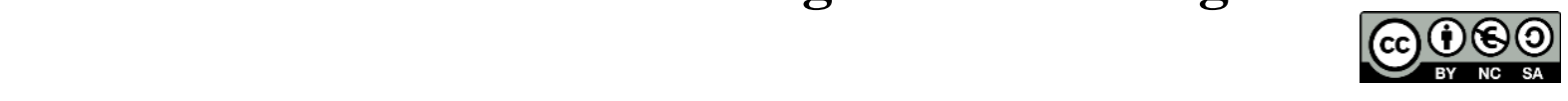

Rate of infiltration of water in agricultural soils.

Edwin Leonardo Sánchez Almeida. ${ }^{1}$, Manolo Alexander Córdova Suárez. ${ }^{2}$, José Geovanny Vega Pérez. ${ }^{3}$, Mentor Leovigildo Córdova Naranjo. ${ }^{4}$, Oscar Eduardo Ruíz Robalino. ${ }^{5}$ \& Tamara de los Ángeles Liger Manzano ${ }^{6 .}$

Recibido: 16-03-2017 / Revisado: 10-05-2017 Aceptado: 11-06-2018/ Publicado: 01-07-2018

\begin{abstract}
.
DOI: https://doi.org/10.33262/cienciadigital.v2i3.183

The efficient location of water resources is an important basis in the migration of nutrients and irrigation of land. Infiltration is the process by which the soil is able to absorb precipitation or irrigation. The unit of measurement of this phenomenon is in millimeters per hour. Through this study it is shown that the silty and fumiduous soils, found in the tisa canton of the tungurahua province in the sierra central zone, have a high level of infiltration, which allows these soils to be propitious for agriculture.
\end{abstract}

Methods: to determine the infiltration level of the limus and fumiferous soil of the tisa canton of tungurahua province, it was applied to a quantitative method, due to the elevation of experimental type values in the types of study objects, analyzing the physical properties of each of them, in addition to their behavior to saturation in the time intervals proposed. An infiltrometer and the horton equation were used to establish the time elapsed since the beginning of the infiltration.

\footnotetext{
1 Indoamerica Technologic University, Ambato-Ecuador, leonardosanchez@uti.edu.ec

2 Department of Food Science and Engineering, Technical University of Ambato, Ambato-Ecuador, jcm1991@ gmail.com

3 G+ Energy-Risks \& Engineering Group, Department of Food Science and Engineering, Technical University of Ambato, Ambato-Ecuador, jg.vega@ uta.edu.ec

4 Faculty of Humanities and Education, Technical University of Ambato, Ambato-Ecuador, mentorlcordova@uta.edu.ec

5 G+ Energy-Risks \& Engineering Group, Department of Food Science and Engineering, Technical University of Ambato, Ambato-Ecuador, oe.ruiz@uta.edu.ec

6 Faculty of Civil and mechanical Engineering, Technical University of Ambato, Ambato-Ecuador, tda.liger@uta.edu.ec
} 
Results: it was determined the time it will take the soil to be totally flooded with water, the height of the infiltration in time and the humidity retained, this procedure was carried out for the silty and fumiduous soil. The infiltration rate in the humid soil is approximately $20 \mathrm{~mm} / \mathrm{h}$. With a humidity of 0.5 .

Conclution: the data raised for the silty soil indicated that the maximum time to reach the height of $38.2 \mathrm{~mm}$ is approximately 90 minutes from the moment of water recovery, that is, it will wait at least 30 minutes after having flooded the crop and have good growing conditions.

Key words: soil, silt, smoke, humidity

\section{Resumen.}

La utilización eficiente de los recursos hídricos es una base importante en la migración de nutrientes y el riego de tierra. La infiltración es el proceso por el cual el suelo es capaz de absorber la precipitación o la irrigación (agua). La unidad de medición de este fenómeno es en milímetros por hora. A través de este estudio se demostró que los suelos limosos y fumíferos, encontrados en el cantón tisaleo de la provincia de tungurahua en la zona sierra centro, poseen un alto nivel de infiltración, lo que permite que estos suelos sean propicios para la agricultura.

\section{Métodos.}

Para la determinación del nivel de infiltración del suelo limos y fumíferos del cantón tisaleo de la provincia de tungurahua, se aplicó un método cuantitativo, debido al levantamiento de valores de tipo experimental en los tipos de suelo objetos de estudio, analizando las propiedades físicas de cada uno de ellos, además de su comportamiento sometidos a la saturación en los intervalos de tiempo planteados. Se utilizó un infiltrómetro y la ecuación de horton para establecer el tiempo transcurrido desde el inicio de la infiltración.

\section{Resultados.}

Se determinó el tiempo que tarda el suelo en inundarse totalmente de agua, la altura de infiltración en el tiempo y humedad retenida, este procedimiento se realizó para el suelo limoso y fumífero. La tasa de infiltración en el suelo humífero es de aproximadamente de $20 \mathrm{~mm} / \mathrm{h}$. Con una humedad de 0,5 .

\section{Conclusión.}

Los datos levantados para el suelo limoso indica que el tiempo máximo para alcanzar la altura de 38,2 $\mathrm{mm}$ es de aproximadamente $90 \mathrm{~min}$ a partir del momento de la riega de 
agua, es decir se deberá esperar al menos 30 min luego de haber inundado el cultivo y tener condiciones óptimas de cultivo.

Palabras claves: suelo, limoso, fumífero, humedad.

\section{Introducción.}

El agua de regadío en la provincia de tungurahua, específicamente en el cantón tisaleo (figura 1) es una de las fuentes más importantes para los agricultores y campesinos. Actualmente las represa de chiquiurco y mula corral son considerados como los de mayor embalse en la provincia, puesto que en ellas se almacena un aproximado de 6 millones de metros cúbicos. Estos embalses sirven para la agricultura y abastecimiento de líquido en los hogares, por lo que, es importante el uso adecuado de este elemento vital (diario la hora 28 mayo 2012).

El canal Huachi Pelileo se sirve de estos embalses ya que toma un volumen aproximado de 31 litros por segundo, la cual es utilizada por cerca de 15000 personas para el riego por inundación en 5380 hectáreas. (Diario la Hora 28 mayo 2012; Rodrigo Cáceres 2012).

Figura 1. Mapa geográfico del cantón Tisaleo en la provincia de Tungurahua.

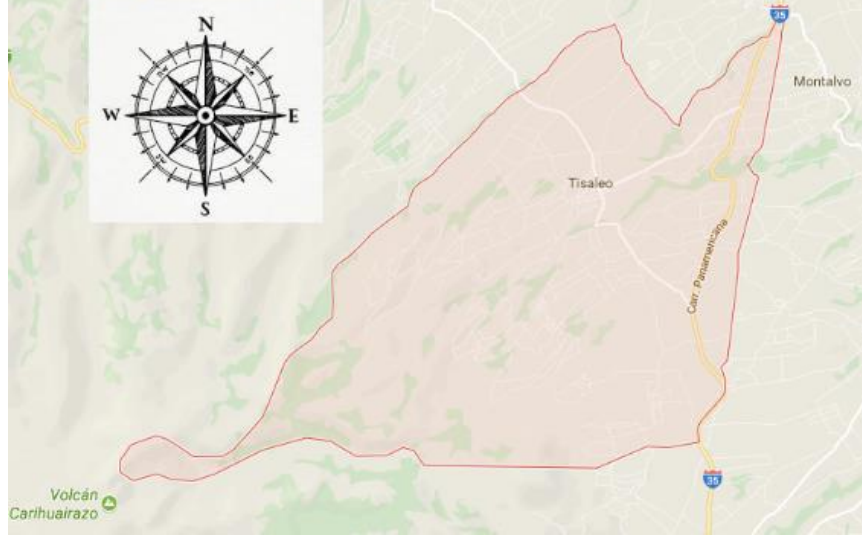

El método de riego por inundación, está basado en hacer circular agua por un canal formado entre dos cúmulos de arena (conocidos como cúmulos de arado) y dejar en reposo al líquido durante unos minutos hasta que se infiltre y se sature en el suelo sin un tiempo preciso que indique que se ha saturado en su totalidad, para luego circular a las demás áreas de cultivo, ver figura 2. 
Figura 2. Vista en corte para método de riego mediante inundación.

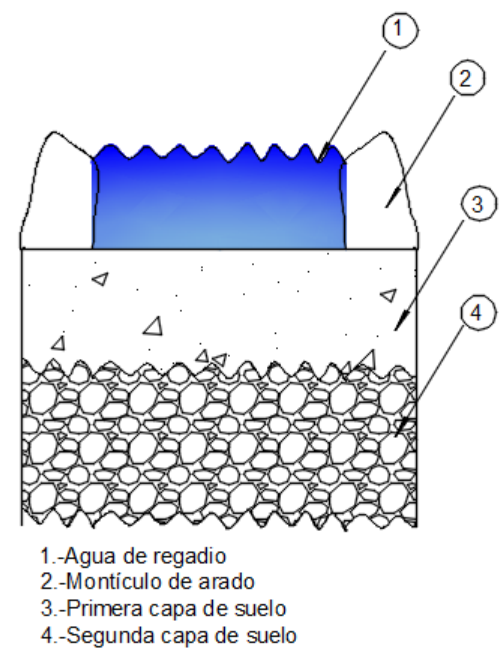

La capacidad de infiltración depende del tipo de suelo (ver figura 3), debido a que se puede tratar de un suelo de tipo limo arcilloso, el cual se caracteriza por su color oscuro por la gran cantidad de humus y restos de compuesto orgánico (desechos vegetales y animales) que lo conforman, además, este tipo de suelo posee una alta capacidad de almacenar agua en su interior (Kaurichev, 1984). El suelo de tipo limoso se caracteriza por tener partículas pequeñas al igual que un suelo de tipo humífero, ambos tipos de suelo filtran el agua con bastante rapidez y son fértiles (Chamizo et al., 2009).

El tipo de suelo arcilloso, presenta una coloración amarillenta, este tipo de suelo retiene alrededor de un 45,5\% de agua_(Russell y Subero, 1934). El suelo de tipo de Turba se caracteriza por su correcto drenamiento del agua, este tipo de suelos son excelentes para ser usados en los cultivos, ya que poseen un $\mathrm{pH}$ acido de 3,35 a 3,95, además, su porosidad permite una mejor retención de la humedad.

Figura 3. Corte en sección de un suelo arenoso.

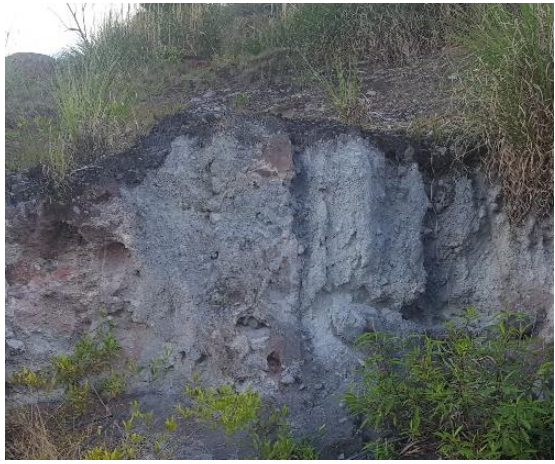


El proceso de infiltración se lo realiza mediante la utilización de un infiltrómetro, que permite obtener datos precisos sobre la cantidad de agua que se requiere para regar un cultivo y el tiempo necesario para alcanzar la saturación necesaria, sin producir el ahogamiento de la planta a través de la porosidad $(n)$ del suelo y el tipo de humedad presente $(\theta)$, esta relación se muestra en la ecuación 1. Además, la porosidad del suelo se determina con la aplicación de la ecuación 2 (Freeze (1979); Freeze y Cherry (1979)).

$$
\begin{aligned}
& \theta=\frac{V_{W}}{V_{T}} \\
& n=\frac{V_{V}+V_{W}}{V_{T}}
\end{aligned}
$$

Las condiciones de humedad presente en el suelo favorecen y desfavorecen la infiltración del agua, por ello de la humedad dependerá el tiempo que se requiera para que el suelo alcance la condición de saturación por acumulación de agua. (Allen, 2006). La capacidad de infiltración (Freeze (1979); Tesoriero y Pankow (1996)) se determina bajo el tipo de porosidad ( $n$ ) del suelo y el tipo de humedad en el suelo $(\theta)$ y se determina bajo la siguiente tabla.

Tabla 1 Porosidad y conductividad hidráulica de suelos.

\begin{tabular}{ccccc}
\hline $\begin{array}{c}\text { Tipo de } \\
\text { suelo }\end{array}$ & $\begin{array}{c}\text { Porosidad } \\
\%\end{array}$ & \multicolumn{2}{c}{$\begin{array}{c}\text { Rango de conductividad } \\
\text { hidráulica }\end{array}$} \\
\hline Arena & $25-50$ & $1 \times 10^{-1}$ & - & $1 \times 10^{-2}$ \\
Limo & $35-50$ & $1 \times 10^{-5}$ & - & 1 \\
Arcilla & $40-70$ & $1 \times 10^{-7}$ & - & $1 \times 10^{-3}$ \\
Grava & $25-40$ & $1 \times 10^{-9}$ & - & $1 \times 10^{-5}$ \\
\hline
\end{tabular}

\section{Materiales y métodos.}

Instrumentos y Técnicas de Recolección de Datos.

El objeto de estudio fueron dos tipos de suelos presentes en el cantón Tisaleo, el suelo fumífero y el suelo limoso. El levantamiento de información se realizó en base a fichas de información y levantamiento de datos conforme a los ensayos experimentales realizados con los tipos de suelo y su capacidad de infiltración en diferentes tiempos.

Los resultados y gráficos se obtuvieron mediante la aplicación del software Excel, el programa estadístico SPSS y la utilización de ecuación de Horton para cálculo de la tasa de infiltración. 


\section{Procedimiento.}

- Se analizaron las dos clases de suelos predominantes en el sector agrícola del cantón Tisaleo

- Se ubicó el infiltrómetro en cada uno de los tipos de suelos en estudio.

- Se aforaron los infiltrómetros hasta la altura que se encuentra graduada (equivalente a 20 litros de agua), tal como se muestra en la figura 4.

- Se evalúo el tiempo en el que el agua baja de nivel según la regla graduada en posición vertical con una escala de 20 centímetros.

- Se determinó el tiempo trascurrido en el que se infiltró el agua en su totalidad, mediante la aplicación de la ecuación de Horton ver ecuación 3.:

$$
f p=f c+(f o-f c) e^{-k t}
$$

Donde:

$\mathrm{fp}=$ Capacidad de infiltración $(\mathrm{mm} / \mathrm{h})$

$\mathrm{k}=$ Factor de proporcionalidad llamado de también de crecimiento

$\mathrm{fc}=$ Capacidad de infiltración final

fo $=$ Capacidad de infiltración inicial (Para $\mathrm{t}=0$ )

$\mathrm{t}$ = Tiempo transcurrido desde el inicio de la infiltración

- Finalmente, transcurrida la infiltración total se cortó el suelo de manera que se apreciaba la sección humedad y se tomaron los datos de posición de humedad en el suelo para su posterior traficación.

Figura 4 Medición de altura de infiltración mediante infiltrómetro.

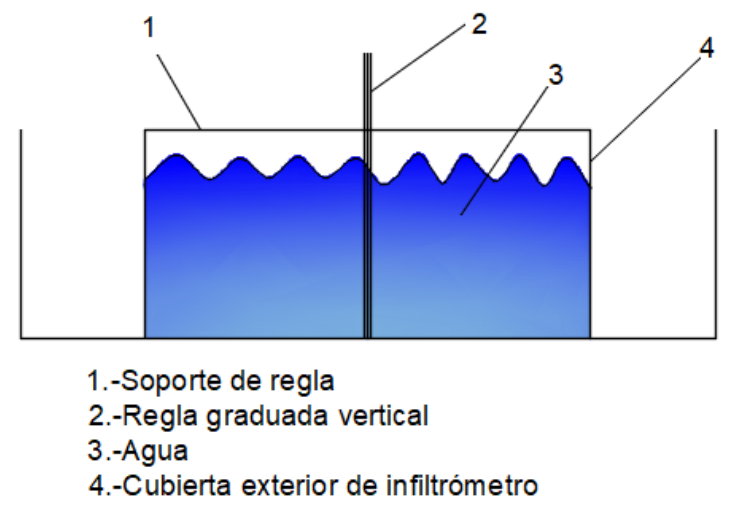




\section{Resultados}

La Tabla 2. muestra el comportamiento del suelo limoso.

Tabla 2 Pruebas de infiltración para diferentes tiempos.

\begin{tabular}{cc}
\hline \multicolumn{2}{c}{ PRUEBAS DE INFILTRACIÓN } \\
\hline Tiempo en min & Altura alcanzada (mm) \\
$\mathbf{5}$ & 5 \\
$\mathbf{1 0}$ & 7 \\
$\mathbf{1 5}$ & 7,5 \\
$\mathbf{2 0}$ & 8 \\
$\mathbf{2 5}$ & 8,2 \\
$\mathbf{3 0}$ & 8,7 \\
$\mathbf{3 5}$ & 10,3 \\
$\mathbf{4 0}$ & 15,1 \\
$\mathbf{4 5}$ & 15,9 \\
$\mathbf{5 0}$ & 16,8 \\
$\mathbf{5 5}$ & 19,16 \\
$\mathbf{6 0}$ & 20,3 \\
$\mathbf{6 5}$ & 24,7 \\
$\mathbf{7 0}$ & 27,1 \\
$\mathbf{7 5}$ & 28,7 \\
$\mathbf{8 0}$ & 32,2 \\
$\mathbf{8 5}$ & 36,4 \\
$\mathbf{9 0}$ & 38,2 \\
\hline
\end{tabular}

Figura 5 Diagrama de tendencia para infiltración. 


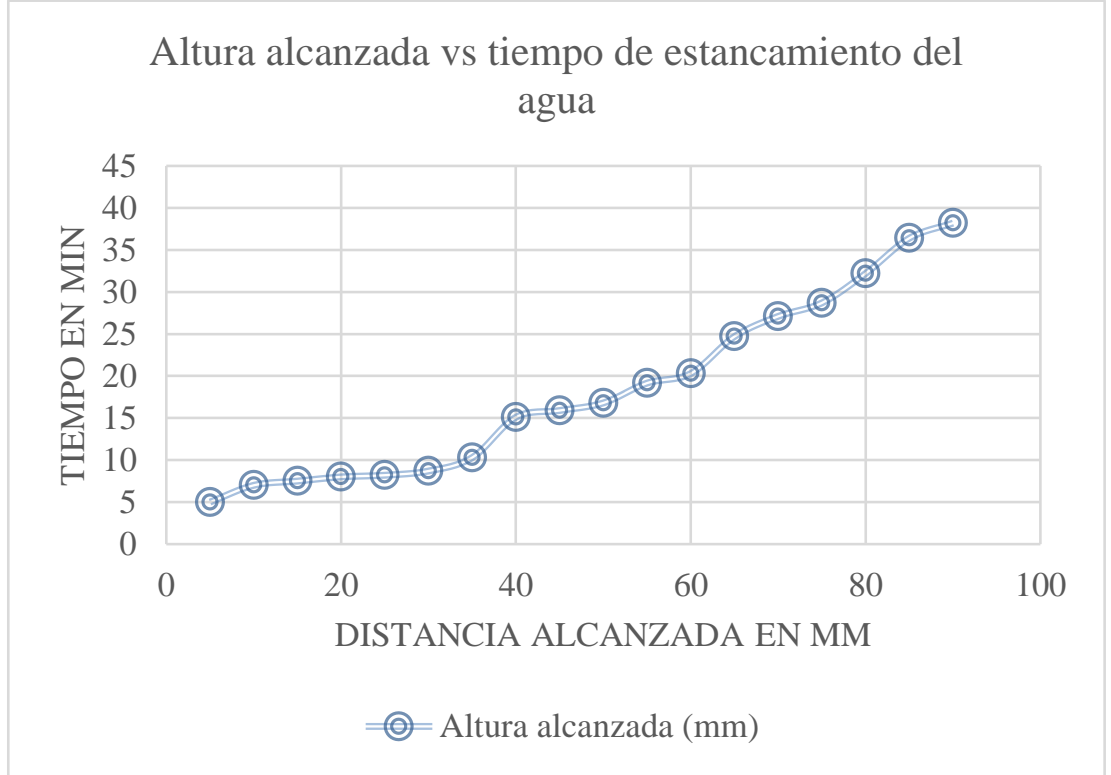

La figura 6 muestra la sección en corte del suelo y su comportamiento de infiltración de agua en el suelo.

Figura 6 Comportamiento de infiltración en suelo humífero

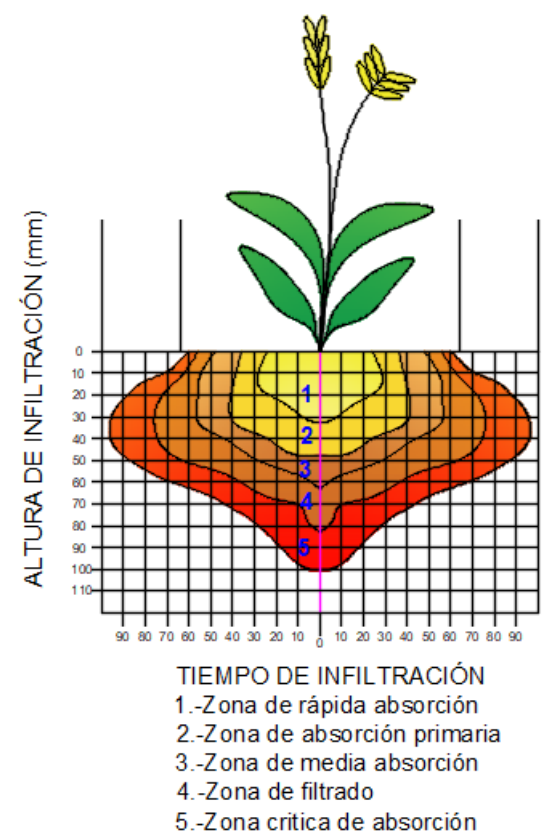




\section{Discusión.}

Los resultados obtenidos muestran un ancho máximo de irrigación de $2 \mathrm{~m}$ en total, con una altura perteneciente a su máximo de irrigación de $32 \mathrm{~mm}$ y una profundidad máxima alcanzada por la humedad de $1 \mathrm{~m}$.

La tasa de infiltración en el suelo humífero es de aproximadamente de $20 \mathrm{~mm} / \mathrm{h}$. con una humedad de 0,5 .

\section{Conclusiones.}

- Los datos levantados para el suelo limoso indica que el tiempo máximo para alcanzar la altura de 38,2 $\mathrm{mm}$ es de aproximadamente $90 \mathrm{~min}$ a partir del momento de la riega de agua, es decir se deberá esperar al menos 30 min luego de haber inundado el cultivo.

- El suelo limoso predomina en la región del cantón Tisaleo con un 90\%, el 10\% restante es una combinación de limo y arena.

- El proceso de inundación es eficaz para cierto tipo de cultivos como lo son la papa, ya que este tubérculo necesita de humedad constante para su desarrollo, pero ineficaz para el trigo, ya que esta no necesita de gran cantidad de agua en el interior de sus raíces para desarrollarse de manera eficaz al igual que otras plantas.

\section{Referencias bibliográficas.}

Allen, R. G. (2006). Evapotranspiración del cultivo: guías para la determinación de los requerimientos de agua de los cultivos (Vol. 56): Food \& Agriculture Org.

Chamizo, A., Ferrera-Cerrato, R., González-Chávez, M., Ortiz-Solorio, C., Santizo-Rincón, J., Varela, L. y Alarcón, A. (2009). Inoculación de alfalfa con hongos micorrízicos arbusculares y rizobacterias en dos tipos de suelo. Terra Latinoamericana, 27(3), 197205.

Freeze, R. A. (1979). GROUNXDWATER.

Freeze, R. A. y Cherry, J. A. (1979). Groundwater, 604 pp: Prentice-Hall, Englewood Cliffs, NJ.

Kaurichev, I. (1984). Prácticas de edafología.

Russell, E. y Subero, S. (1934). Condiciones del suelo y crecimiento de las plantas.

Tesoriero, A. J. y Pankow, J. F. (1996). Solid solution partitioning of $\mathrm{Sr} 2+$, Ba 2+, and Cd 2+ to calcite. Geochimica et Cosmochimica Acta, 60(6), 1053-1063. 
Para citar el artículo indexado.

Sánchez E., Córdova M., Vega J., Córdova M., Ruíz O. \& Liger T. (2018). Tasa de infiltración de agua en suelos agrícolas . Revista electrónica Ciencia Digital 2(3), 662-671. Recuperado http://cienciadigital.org/revistacienciadigital2/index.php/CienciaDigital/article/view/183/162

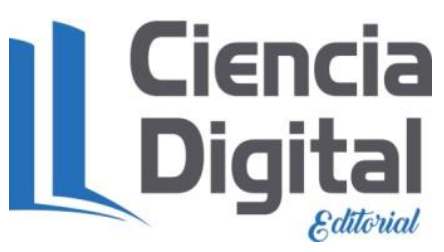

El artículo que se publica es de exclusiva responsabilidad de los autores y no necesariamente reflejan el pensamiento de la Revista Ciencia Digital.

El articulo queda en propiedad de la revista y, por tanto, su publicación parcial y/o total en otro medio tiene que ser autorizado por el director de la Revista Ciencia Digital.
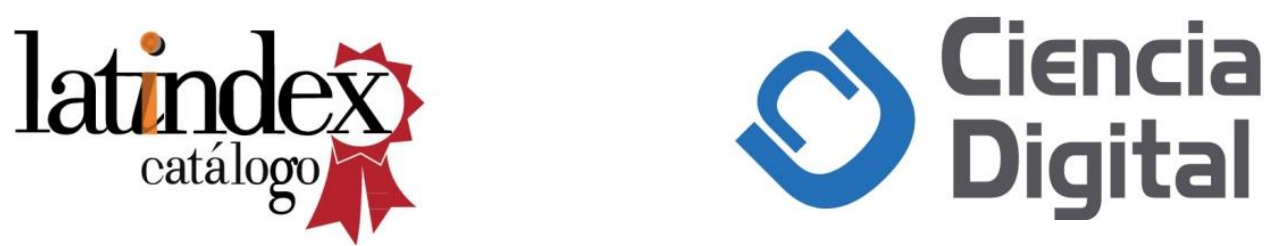